\title{
Comparison of Algorithms for Wind Parameters Extraction From Shipborne X-Band Marine Radar Images
}

\author{
Ying Liu, Student Member, IEEE, Weimin Huang, Senior Member, IEEE, Eric W. Gill, Senior Member, IEEE, \\ Dennis K. Peters, Senior Member, IEEE, and Raul Vicen-Bueno
}

\begin{abstract}
In this paper, curve-fitting and intensity-levelselection (ILS)-based algorithms for wind parameter extraction from shipborne $\mathbf{X}$-band nautical radar images are investigated. First, to exclude the rain cases and low-backscatter images, a data quality control process is designed for both algorithms. An additional process is then introduced for the ILS-based method to improve the accuracy of wind measurements, including the recognition of blockages and islands in the temporally integrated radar images. For the low sea states, a dual-curve-fitting is proposed. These wind algorithms are tested using radar images and shipborne anemometer data collected on the east coast of Canada. It is shown that the dual-curve-fitting algorithm produces improvements in the mean differences between the radar and the anemometer results for wind direction and speed of about $5.7^{\circ}$ and $0.3 \mathrm{~m} / \mathrm{s}$, respectively, under sea states with significant wave height lower than $2.30 \mathrm{~m}$. Also, a harmonic function that is least-squares fitted to the selected range distances vector as a function of antenna look direction is applied. Compared with the original ILS-based algorithm, the modified procedure reduces the standard deviation for wind direction and speed by about $4^{\circ}$ and $0.2 \mathrm{~m} / \mathrm{s}$, respectively. Finally, the performance of these two modified methods are compared.
\end{abstract}

Index Terms-Shipborne nautical radar, wind speed and direction, X-band.

\section{INTRODUCTION}

$\mathbf{S}$ EA SURFACE wind information is important for marine navigation, and anemometers have been widely installed on ships for this purpose. However, anemometer measurements may be negatively impacted by the sensor's height and the ship's motion and structure. Even at well-exposed locations on

Manuscript received April 24, 2014; revised June 20, 2014; accepted September 01, 2014. Date of publication September 24, 2014; date of current version February 09, 2015. The work of W. Huang was supported by the Natural Sciences and Engineering Research Council of Canada (NSERC) under Grant NSERC 402313-2012. The work of E. Gill was supported in part by the NSERC under Grant NSERC 238263-2010 and in part by the Atlantic Innovation Fund Award. The work of E. Gill and W. Huang was also supported by the Department of Innovation, Business and Rural Development of Newfoundland and Labrador under Grant 30-10921-008.

Y. Liu, W. Huang, E. W. Gill, and D. K. Peters are with the Faculty of Engineering and Applied Science, Memorial University of Newfoundland, St. John's, NL A1B 3X5, Canada (e-mail: 1y3562@mun.ca; weimin@mun.ca; ewgill@mun.ca; dpeters@mun.ca).

R. Vicen-Bueno is with the Department of Signal Theory and Communications, Superior Polytechnic School, University of Alcala, Madrid 28805, Spain (e-mail: raul.vicen@uah.es).

Color versions of one or more of the figures in this paper are available online at http://ieeexplore.ieee.org.

Digital Object Identifier 10.1109/JSTARS.2014.2357426 the ship, the anemometer may have a wind speed measurement error of up to $10 \%$ [1]. This is one of the main reasons why efforts are now being made to retrieve wind information from nautical radar images. Moreover, compared to traditional in situ measurements, radar-based wind measurements have an additional advantage that they are independent of the sensor's height and motion [2].

$\mathrm{X}$-band radar backscatter from the ocean surface is mainly due to its interaction with the small-scale roughness generated by local wind [3]. It has been shown that the normalized radar cross section (NRCS) strongly depends on wind speed [3], [4] and direction [4], [5]. For Horizontally polarized (HH-polarized) radar at grazing incidence, the NRCS has only one peak in the upwind direction and is minimum in the crosswind direction [4], [5], and it is an exponential function of wind speed [6]. Researchers have attempted to retrieve wind information from nautical radar images [7]-[9]. Dankert et al. [7] determined wind direction from quasi-stationary wind streaks, and extracted wind speed from the temporally integrated radar images and the retrieved wind direction. However, this approach requires an additional processing step, such as geocoding, in order to be applied to shipborne radar data because, as a result of the platform's horizontal motion, wind streaks are difficult to extract [10]. Also, a $180^{\circ}$ directional ambiguity exists in the wind direction results but it can be removed by extracting the movement of wind gusts visible in the radar image sequence [6]. Recently, Lund et al. [8] and Vicen-Bueno et al. [9] developed methods which are independent of platform movement. By utilizing the radar backscatter intensity dependence on the upwind direction, Lund et al. [8] proposed a least-squares curve-fitting technique to identify the upwind peak and an empirical third-order polynomial to calculate wind speed. Vicen-Bueno et al. [9] developed an effective backscatter intensity-level-selection (ILS) algorithm based on temporal integration and spatial smoothing as well as an empirical third-order polynomial geophysical model function (GMF) to derive wind direction and speed. The latter two algorithms can be applied to radar data collected from a moving ship, and thus are considered in this paper. However, modifications need to be made for these two algorithms. In the curve-fitting process, although the data in the directions due to blockage were excluded, the low-intensity data in many azimuthal directions due to low sea states rather than the obstruction by ship structures may be retained, and the result becomes 
TABLE I

DATA INFORMATION

\begin{tabular}{c|c|c|c|c|c|c}
\hline $\begin{array}{c}\text { Dataset } \\
\text { no. }\end{array}$ & Antenna & $\begin{array}{c}\text { Antenna rotation } \\
\text { speed (RPM) }\end{array}$ & Data collecting time & $\begin{array}{c}\text { Number of } \\
\text { images }\end{array}$ & $\begin{array}{c}\text { Number of } \\
\text { rain cases }\end{array}$ & $\begin{array}{c}\text { Number of } \\
\text { low-backscatter images }\end{array}$ \\
\hline 1 & Decca & 28 & $\begin{array}{c}\text { Nov. 26, 23:45-Nov. 28, 04:04 } \\
\text { Nov. 28, 11:16-Nov. 29, 12:06 }\end{array}$ & 49182 & 9924 & 1832 \\
\hline 2 & Furuno & 40 & Dec. 01, 12:09-Dec. 04, 12:02 & 69088 & 992 & 8337 \\
\hline 3 & Decca & 28 & Nov. 29, 01:12-Nov. 29, 05:31 & 3872 & 0 & 224 \\
\hline 4 & Furuno & 40 & Oct. 29, 17:46-Oct. 29, 18:46 & 992 & 0 & 0 \\
\hline
\end{tabular}

less accurate. Under these circumstances, a second stage of curve-fitting is proposed to reduce the wind estimation error. On the other hand, since the obstruction of the radar field of view or appearance of islands was not considered in [9], to reduce the wind measurement errors in this case, an additional process for recognizing the view of blockages and islands as well as a similar curve-fitting technique are designed for the intensity-level-selection algorithm.

This paper represents an extension of the work appearing in [11] and [12] and is organized as follows. In Section II, the $\mathrm{X}$-band radar data and the reference wind data are described. Section III introduces the data quality control procedures, including the recognition of rain and low-backscatter images. In Section IV, the curve-fitting, the ILS-based wind algorithms [9], and the associated modifications are introduced. The comparisons of wind direction and speed with in situ measurements are discussed in Section V. Finally, Section VI contains a summary and reiterates the main conclusion of this work.

\section{DATA OVERVIEW}

The data used for testing were provided by Defence Research and Development Canada (DRDC). The marine radars utilized in the experiment are standard HH-polarized shipborne Decca and Furuno nautical radars which operate at $9.41 \mathrm{GHz}$. The Decca and Furono radars cover $360^{\circ}$ in azimuth with approximate beam width of $2^{\circ}$ and $1.9^{\circ}$, respectively. The radar range extends to $2160 \mathrm{~m}$ (starting at $240 \mathrm{~m}$ in the near range) with a range resolution of $7.5 \mathrm{~m}$. The radar was connected to a Wave Monitoring System II (WaMoS II) [13]. The system digitizes the radar backscatter intensities by azimuth-range bin and scales data into 8-bit unsigned integers $([0,255])$. Sets of 32 radar images are combined into one file, and the file index reveals the start time of each file.

The information of the data used in this paper is listed in Table I. The collection times are shown in standard local time coordinates. It should be noted that the first three datasets were collected during the same sea trial but Dataset 4 was collected from another experiment. The shipborne anemometer and marine radar data were all collected on the Canadian Navy research ship CFAV Quest [14] approximately 150 miles from the coast of Halifax $\left(42^{\circ} \mathrm{N}, 62^{\circ} \mathrm{W}\right)$ in late October, late November and early December, 2008 [15]. Two anemometers were installed in the port and starboard side, respectively. Only the data measured by the starboard-side anemometer are used because the wind results from these two anemometers are very similar. Since the heights of the anemometers are not available, the wind data could not be converted to the corresponding values at $10 \mathrm{~m}$ above the water. The anemometer wind data were affected by the ship's motion. Thus, the reference wind speed and direction are calculated by removing the ship's motion, for which only the ship velocity over ground is considered here. Dataset 3 is a 4-h subset of Dataset 1 which includes the data under low sea states, and it is used to compare the performance of dual-curve-fitting and single-curve-fitting under low sea states. Dataset 4 is a 1-h dataset extracted from a 2-day long experiment in late October during which data were collected by a Furuno radar. This dataset is employed to test the modified ILS-based algorithms with respect to island recognition and removal.

\section{QuAlity CONTROL}

Before using the radar data to determine the upwind direction and wind speed, a basic data quality control procedure similar to that in [8] was undertaken for each image by analyzing the radar backscatter intensity histogram and zero-pixel percentage. The procedure includes the recognition of rain and low-backscatter images.

\section{A. Rain Recognition}

It has been observed that rain affects the radar backscatter through volume scattering and attenuation by raindrops in the intervening atmosphere and sea surface roughness change resulted by rain impinging on the ocean [16], [17]. For radar images collected during rainfall, the NRCS depends on the rainfall rate, drop size, radar frequency, and polarization [16]-[18]. However, in this case, the NRCS may be enhanced or diminished, depending on the specific combination of these four factors. According to [8], due to the strong impact of rain on the number of pixels with zero intensity in X-band nautical radar images, the zero-pixel percentage (ZPP, i.e., ratio, expressed as a percentage, of the number of image pixels with zero intensity to the overall number of pixels) was identified as a quality control parameter to determine the presence of rain. For the data presented in this paper, pixels with gray scale intensity lower than 5 were considered as zero-intensity. This value should be adjusted for data with a different gray scale depth. It is worth mentioning that the blockage portion in the images should be excluded for the ZPP calculation. Moreover, it should be noted that the thresholds of the ZPP for recognizing rain cases for the Decca and Furuno radars are different. The difference may be explained by the possibly different shadowing effects due to different antenna heights. In Dataset 1, when the ZPP of the image was below $10 \%$, the image was regarded as contaminated by rain, and was excluded from the process of establishing the third-order polynomial model functions of wind speed for both methods. For Dataset 2, the ZPP threshold is $20 \%$. 


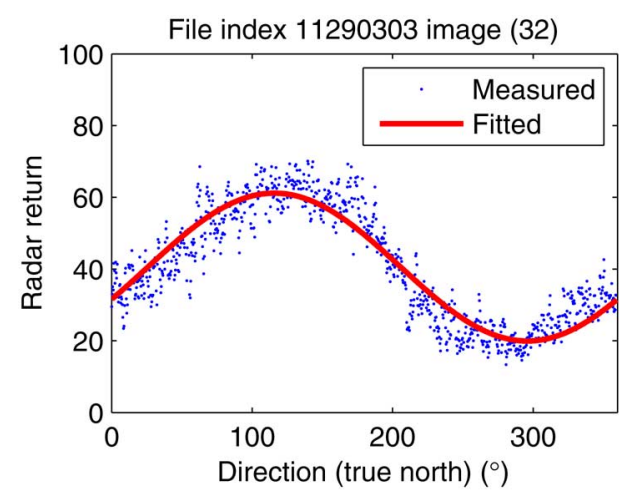

Fig. 1. Range-averaged radar backscatter gray scale intensity as a function of antenna look direction for image collected at 03:03, November 29, 2008.

Examples of the corresponding radar backscatter images and their histograms in the presence of rain can be found in [19].

\section{B. Low-Backscatter Image Recognition}

As a result of too-low wind speed or unknown system errors, some images may appear almost completely black with little or no wave signature. These images are referred to as lowbackscatter images. A parameter, referred to as the low-clutter direction percentage, is used for specifying low-backscatter images. If the ZPP of an azimuthal direction is higher than $40 \%$ for the Decca or $80 \%$ for the Furuno radar (empirical and also varies with systems), the direction is considered to be a lowclutter (including blockage) direction. Then, if the low-clutter direction percentage (LCDP, i.e., the number of low-clutter directions divided by the number of pulses) of an image is higher than $90 \%$, the image is recognized as a low-backscatter image and will not be used for wind retrieval.

\section{Wind PARAmeter EXTRaction Algorithms}

\section{A. Curve-Fitting-Based Wind Algorithms}

1) Single-Curve-Fitting: For HH-polarized X-band radars operating at grazing incidence, it is known that the radar backscatter intensity has only one peak in the upwind direction [4], [5], but a second peak appears in the downwind direction at moderate incidence angles [20], [21]. To obtain the specific dependence on antenna look direction, radar data for the Decca and Furuno systems are averaged over ranges $450-1500 \mathrm{~m}$ and 375-975 m, respectively, for each azimuthal direction $\theta$ and are then curve fitted using a cosine square function to give the range-averaged radar backscatter gray scale intensity as [8]

$$
\sigma_{\theta}=a_{0}+a_{1} \cos ^{2}\left(0.5\left(\theta-a_{2}\right)\right)
$$

where $a_{0}, a_{1}$, and $a_{2}$ are the regression parameters. An example of curve-fitting is shown in Fig. 1. The blue dots represent the measured averaged backscatter intensity, and the best-fit curve is shown by red line. For each individual radar image, the wind direction can be retrieved from the upwind backscatter peak using the model function of (1). The upwind peak direction is given by the regression parameter $a_{2}$. This corresponds to the peak of the best-fit curve, as discussed in [8]. This method

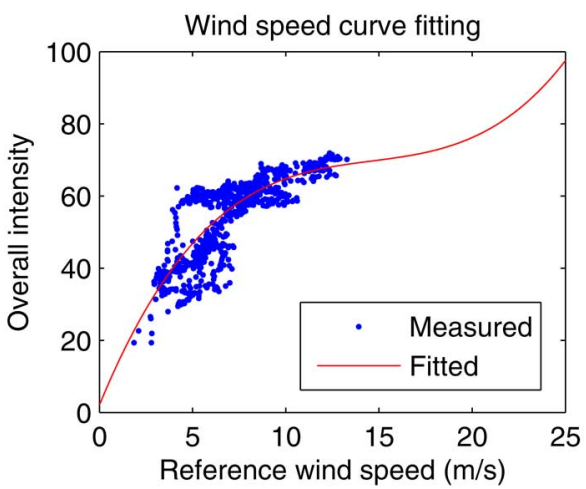

Fig. 2. Scatter plot showing the wind speed from anemometer, corresponding radar backscatter intensity, and the best-fit curve based on a third-order polynomial function for Decca data acquired during November 28-29, 2008.

works well even when some sections of the radar field of view are masked. Based on the radar backscatter intensity dependence on wind speed, an empirical third-order polynomial can be derived using the average radar backscatter intensity and the reference wind speed measured by other sensors (e.g., an anemometer). Then, radar wind speed results can be retrieved from the average intensity value. The average radar backscatter intensity $\sigma_{w S p d}$ is calculated as [8]

$$
\sigma_{w S p d}=\frac{1}{2 \pi} \int_{0}^{2 \pi}\left(a_{0}+a_{1} \cos ^{2}\left(0.5\left(\theta-a_{2}\right)\right)\right) d \theta .
$$

Fig. 2 shows a scatter plot of the anemometer-measured wind speed and the corresponding radar average backscatter intensities $\sigma_{w S p d}$. The best-fit curve indicated by the red line is derived using a least-squares method based on a third-order polynomial function as in [8].

2) Dual-Curve-Fitting: To retrieve wind direction from a single image, the radar backscatter intensities from the nonobstructed antenna look directions are averaged over range to perform the curve-fitting. The data in the directions due to blockage were excluded. However, for some images, in many azimuthal directions, a majority of the pixels may have a very low intensity [see Fig. 3(a)]. These dark regions are not due to the obstruction by ship structures but probably result from low sea states. These low-intensity data are retained in the curve-fitting process. Unfortunately, as shown in Fig. 3(b), in these low-intensity azimuthal directions, the single-curvefitting result is not ideal. In this case, a dual-curve-fitting may be implemented. The technique involves using the data at angles of $60^{\circ}$ to the left and right of the first-guess upwind direction on which the curve-fitting is repeated. Since the data in the directions around the direction with maximum backscatter (upwind) usually have higher signal-to-noise ratio (SNR) than the data in other directions, using this portion of the data for curve-fitting leads to a more robust result. The direction range chosen for the second curve-fitting should be wide enough to include the upwind direction. However, if this range is too large, performance may be affected because data with low SNR may be included. If the range is chosen to be too narrow, the upwind peak may be missed and the fitting result may not be good due to a small number of data points. The $\pm 60^{\circ}$ range is chosen 

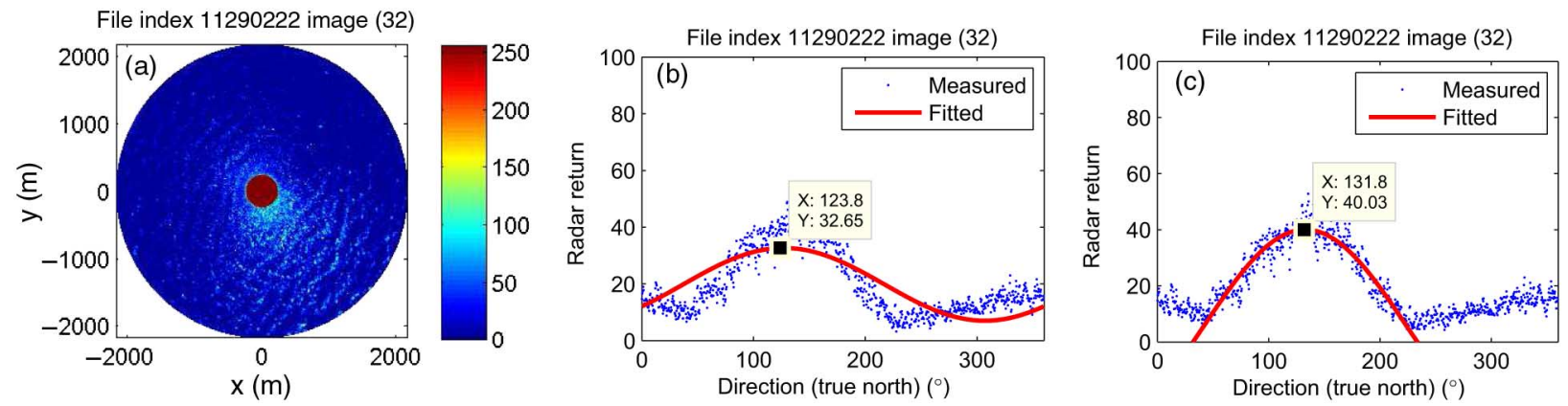

Fig. 3. Example of the Decca radar data from Dataset 1 (collected at 02:22, November 29, 2008): (a) backscatter image; (b) range-averaged backscatter intensity as a function of antenna look direction using single-curve-fitting; and (c) range-averaged backscatter intensity as a function of antenna look direction using dual-curve-fitting.
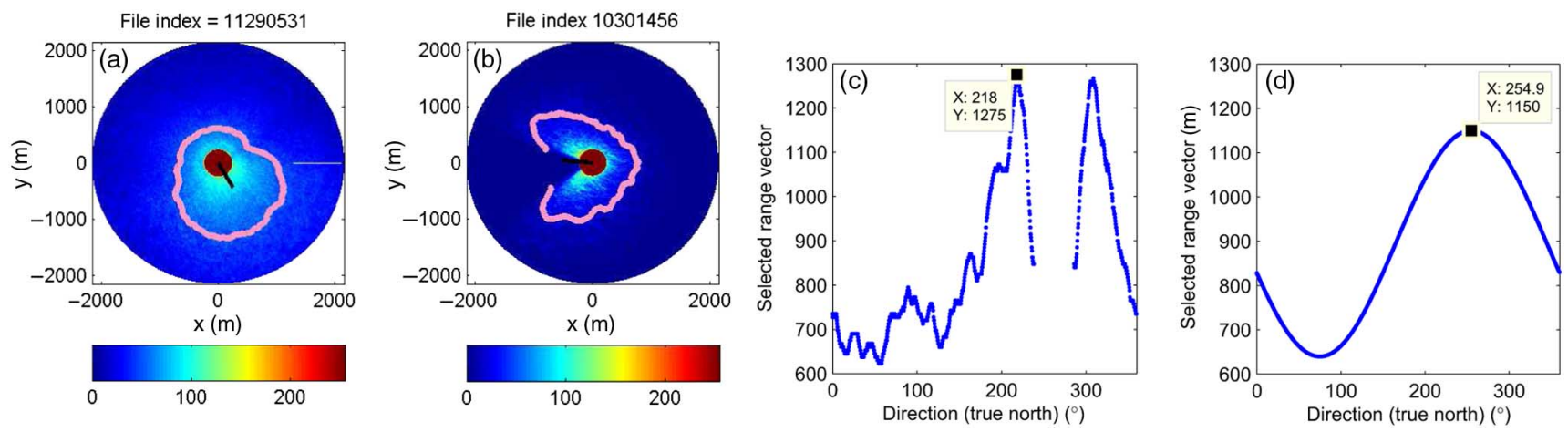

Fig. 4. (a) An integrated image without blockage from Dataset 1 (Decca radar). The black bar at the center shows the anemometer-measured wind direction. (b) An integrated image with blockage from Dataset 4 (Furuno radar). (c) The selected range distances vector without curve-fitting referred to (b). (d) Curve-fitted selected range distances vector referred to (b). The short line in (a) and (b) indicates the anemometer-measured wind direction.

since it yields satisfactory results. In the example shown in Fig. 3(c), the dual-curve-fitting results in an improvement of $8^{\circ}$, as compared to that obtained using a single-curve-fitting. It should also be noted that, when the dual-curve-fitting is applied, the data in the directions with nonpositive fitted values are excluded for evaluating the average radar backscatter intensity $\sigma_{w S p d}$.

\section{B. ILS-Based Wind Algorithms}

1) Original ILS: The original ILS-based method in [9] includes integration and smoothing of radar images, intensitylevel selection, and wind direction and speed estimation. For the data presented in this paper, since each B-scan image (i.e., in polar coordinates) has 288 range bins (including 32 bins associated with the dead range) but a different number of pulses, the radar images cannot be directly summed. Interpolation to a standardized grid is implemented to generate B-scan images with a uniform size of 1024 (pulses) $\times 288$ (ranges). The temporal integration is performed on the entire sequence of files (many hours of data) by utilizing a moving average of every 32 radar images with a shift of four images. As in [9], each time, average result is further smoothed over five range cells (two leading and two lagging with the result being assigned to the central cell) and an azimuthal extent of $5^{\circ}$. As addressed below, each integrated image is assigned a certain intensity level and associated range distances vector, from which the wind information can be extracted. The steps are as follows. a) Intensity-Level Selection: The general technique is given in [9] and the particulars of its present application follow. By analyzing the histograms of the integrated radar images, the predefined levels set for both radars in our study were chosen as $L_{1}=5, L_{2}=10, \ldots$, and $L_{25}=125$, on a scale of 0 to 255. In the integrated image, for each predefined intensity level $L_{i}(i=1,2, \ldots, 25)$, the first range distance where the backscatter intensity is smaller than $L_{i}$ is sought in each azimuthal direction. All the range distances associated with each $L_{i}$ are then stored as elements of the range distances vector $r_{i}$. From these vectors, the one with the lowest $L_{i}$, for which all elements are greater than the inner distance boundary [the near-range distance boundary plus a guard range $(7.5 \times 32 \mathrm{~m}+75 \mathrm{~m}=315 \mathrm{~m})$ ], is selected. After the first eight integrated images, only the last selected level $L_{i}$ and its two adjacent intensity levels $L_{i+1}$ and $L_{i-1}$ need to be used to obtain three corresponding range distances vectors. Among these three vectors, the one satisfying the inner distance boundary and having the lowest intensity level is used for wind direction retrieval and is referred to as the retrieval range distances vector. In the case of Fig. 4(a), the selected intensity level from all the predefined levels is $L_{i}=60$. Its smoothed range distances vector for $L_{i}=60$ is plotted as a magenta ring.

b) Wind Direction and Speed Retrieval: In [9], the wind direction is determined to be along the azimuth in which the maximum of the retrieval range distances vector is located. It is also assumed that wind speed $u_{0}$ is related to the maximum 

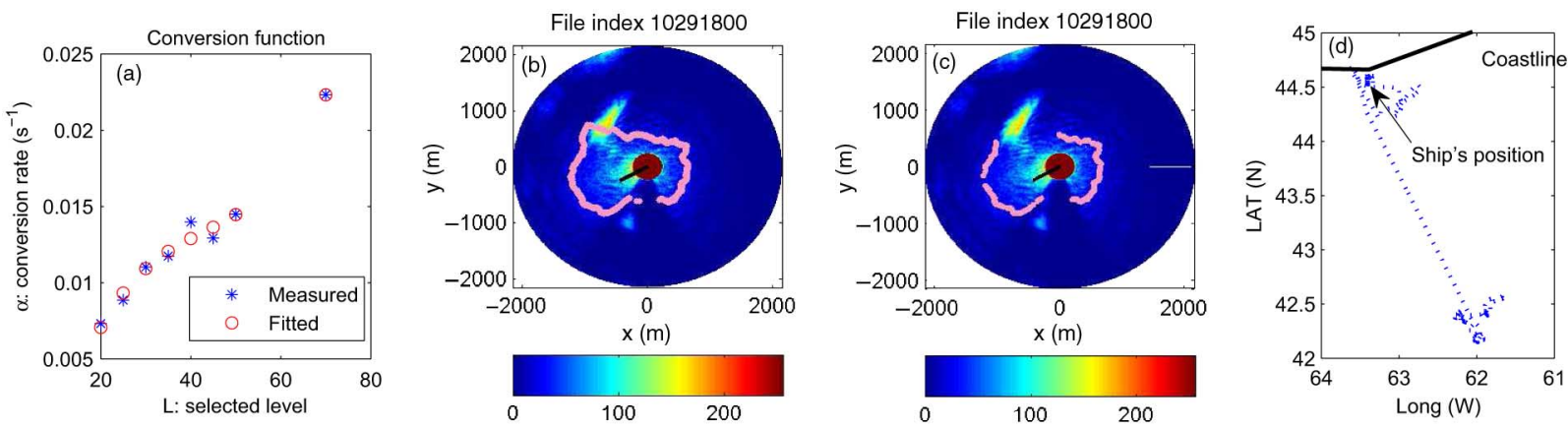

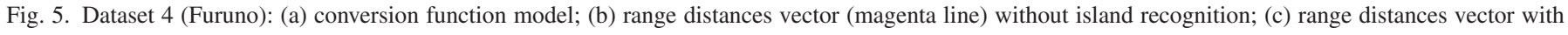
island recognition; and (d) map with ship track.

of the retrieval range distances vector corresponding to the selected intensity level $L_{i}$ as

$$
u_{0}=\alpha_{i} \times \max \left\{r_{i}\right\}
$$

where $\max \left\{r_{i}\right\}$ is the maximum of the retrieval range distances vector and $\alpha_{i}$ represents the conversion rate corresponding to the selected intensity level $L_{i}$. However, the relationship between $\alpha_{i}$ and $L_{i}$ is nonlinear and can be fitted by the third-order polynomial function [9]

$$
\alpha_{i}=\beta_{3} L_{i}^{3}+\beta_{2} L_{i}^{2}+\beta_{1} L_{i}+\beta_{0}
$$

in which the parameters $\beta_{j}(j=0,1,2,3)$ can be determined by least-squares fitting. The fitting is implemented based on the obtained $L_{i}$ and $\alpha_{i}$ that is calculated from (3) using in situ wind speed and $\max \left\{r_{i}\right\}$. Fig. 5(a) shows the conversion rate function obtained from Dataset 4. Red dots and blue stars, respectively, represent the fitted and the calculated $\alpha_{i}$. After the polynomial function is obtained, wind speed is calculated from the maximum range distance associated with $L_{i}$ and the selected $\alpha_{i}$.

2) Modified ILS: The following modifications have been made to improve the robustness of the original ILS algorithm.

a) Additional Constraint: An additional constraint is used to reduce the possibility of wind direction estimation error caused by the noise in the very far range. In addition to being greater than the inner distance boundary $(315 \mathrm{~m})$, all the elements of the retrieval range distances vector $r_{i}$ should also be smaller than the outer distance boundary [far-range distance boundary minus a guard range $(7.5 \times 288-300=1860 \mathrm{~m})$ ]. While searching for this range distances vector, it should be noted that for each azimuthal direction, the first range where the intensity is smaller than the preselected level is used rather than the last index where the intensity is greater. This is done in order to avoid possible interference caused by an island in the far range.

b) Blockage Recognition: For the data considered in this paper, shadowing of the radar field of view exists in many images [see the example in Fig. 4(b)]. The broken part of the selected range distances vector (marked as an open magenta ring) shows the blocked area. It should suffice to determine the blockage once for each radar station if the blockage is due to a stable ship structure. However, the real-time monitoring of blockage will allow the algorithm to be applied to a broader class of conditions which accounts for the fact that there may be blockage induced by severe weather or when operators deliberately or otherwise block data from a particular sector, but in no particular pattern. In such cases, each integrated image should be subjected to real-time blockage recognition. If the percentage of zero pixels is higher than $20 \%$ in a direction, this direction is considered to be blocked and the data located in this part of the integrated image are discarded. Otherwise, the ILS algorithm may fail to find a qualified predefined level.

c) Curve-Fitting: After discarding the obstructed data, problems may still exist, as shown in Fig. 4(c). If the wind direction is aligned with the blocked angles, the abovementioned algorithm will not produce the correct direction. Inspired by the work in [8], a harmonic function, similar to (1)

$$
r_{i}(\theta)=b_{0}+b_{1} \cos ^{2}\left(0.5\left(\theta-b_{2}\right)\right)
$$

was introduced to fit the selected range distances vector [see Fig. 4(b)-(d)]. In (5), $b_{0}, b_{1}, b_{2}$ are coefficients to be determined by curve-fitting. Usually, the locus of the tip of the range distances vector forms a cardioid. This property can be utilized to avoid multiple maxima situations and improve the accuracy of wind direction determination. In Fig. 4(c), the range distances vector maximum is $218.0^{\circ}$ and is corrected by curve-fitting to $254.9^{\circ}$ (with respect to true North) as shown in Fig. 4(d), whereas the corresponding anemometer-measured wind direction is $275.2^{\circ}$ [see Fig. 4(b)]. Therefore, the gap in the original range distances vector plot can be filled.

d) Island Recognition: In Fig. 5(b), a large object (yellow area in the top left corner) appears in the integrated image. By observing the ship's track, indicated by the blue dots in Fig. 5(d), it was found that at the time of Fig. 5(b), the ship position to which the arrow points in Fig. 5(d) is very close to the coastline (black line). Thus, the object is probably a small island. In the wind direction algorithm, the first range index where the intensity is smaller than the pre-selected intensity level $L_{i}$ is chosen. It can be inferred that if the island is located in the far range, the island's influence can be automatically neglected. However, when the island is close to the ship, estimation of the maximum range for $L_{i}$ and the wind direction may be incorrect [see Fig. 5(b)]. In this case, by utilizing the attenuation property of the radar backscatter intensity along range, if an intensity bump is detected in an azimuthal direction, it is assumed that the island is located in that direction and 
TABLE II

Wind SPEED AND Direction RETRIEVAL ERror STATISTICS: BiAs AND STD

\begin{tabular}{|c|c|c|c|c|c|}
\hline \multirow[t]{2}{*}{ Dataset no. } & \multirow[t]{2}{*}{ Algorithm } & \multicolumn{2}{|c|}{ Wind direction error } & \multicolumn{2}{|c|}{ Wind speed error } \\
\hline & & $\operatorname{Bias}\left({ }^{\circ}\right)$ & STD $\left({ }^{\circ}\right)$ & $\operatorname{Bias}(\mathrm{m} / \mathrm{s})$ & STD $(\mathrm{m} / \mathrm{s})$ \\
\hline \multirow[t]{4}{*}{1} & Single curve fitting & 1.5 & 15.3 & 0.4 & 1.4 \\
\hline & Dual curve fitting & 1.8 & 14.9 & 0.4 & 1.4 \\
\hline & ILS & 3.0 & 20.8 & 1.2 & 1.5 \\
\hline & Modified ILS & 1.7 & 15.9 & 0.6 & 1.4 \\
\hline \multirow[t]{4}{*}{2} & Single curve fitting & 1.2 & 17.0 & 0.3 & 2.0 \\
\hline & Dual curve fitting & 1.1 & 16.6 & 0.2 & 1.9 \\
\hline & ILS & 3.8 & 21.2 & 1.1 & 1.5 \\
\hline & Modified ILS & 0.8 & 16.3 & 0.6 & 1.3 \\
\hline \multirow[t]{4}{*}{3} & Single curve fitting & 1.7 & 8.3 & 0.8 & 0.9 \\
\hline & Dual curve fitting & 1.1 & 6.6 & 0.7 & 0.8 \\
\hline & ILS & 4.4 & 10.3 & 0.3 & 0.8 \\
\hline & Modified ILS & 1.9 & 9.3 & 0.3 & 0.5 \\
\hline \multirow[t]{4}{*}{4} & Curve-fitted ILS without island recognition & 14.1 & 18.8 & 0.2 & 1.3 \\
\hline & Curve-fitted ILS with island recognition & 1.3 & 11.8 & 0.1 & 1.0 \\
\hline & Single curve-fitting without island recognition & 12.6 & 19.7 & 0.8 & 1.8 \\
\hline & Single curve-fitting with island recognition & 4.2 & 16.5 & 0.7 & 1.6 \\
\hline
\end{tabular}

the corresponding radar data will be excluded from curve-fitting [see Fig. 5(c)]. The built-in function, findpeaks, in MATLAB is used here to detect peaks or extrema of the intensity sequence for each azimuthal direction. Geographic data may be used to replace the island recognition scheme if such data are available.

\section{RESUlts}

The single-curve-fitting, dual-curve-fitting, original ILS, and modified ILS-based algorithms described above were applied to the quality-controlled Decca and Furuno radar data and the results were compared to the reference data measured by a shipbased anemometer (with the ship's motion removed). Since it must take a finite amount of time for the ocean roughness to react to wind change, unlike anemometer, the radar may not pick up short-time scale wind variations. As is commonly done, 10-min averaging was applied to both the anemometer and radar wind results before computing the comparison statistics. The same data, excluding rain-contaminated and lowbackscatter cases, were used to determine the wind speed calibration functions for both the curve-fitting and ILS-based methods. Since the radars for Datasets 1 and 2 are different, wind speed models were developed for each of the two radars. The data over the periods of 11:16 Nov. 28-11:58 Nov. 29 in Dataset 1 and 12:08 Dec. 1-21:20 Dec. 2 in Dataset 2 were used for training for the Decca and Furuno radars, respectively. The wind speed standard deviations (STD) of the Decca training and validation (nontraining) data are 1.2 and $1.7 \mathrm{~m} / \mathrm{s}$, respectively; whereas, for the Furuno radar, they are 1.6 and $2.0 \mathrm{~m} / \mathrm{s}$, respectively. Thus, the statistics do not differ significantly between the training and validation subsets. The statistics of the wind results for each entire dataset utilizing the four algorithms are shown in Table II.

\section{A. Single-Versus Dual-Curve-Fitting}

Fig. 6 shows the comparison of the radar-derived wind velocities using single- and dual-curve-fitting methods with the anemometer-measured results based on Dataset 3 collected during 01:12 to 05:31, November 29, 2008. It can be seen that the radar results from both methods agree well with the anemometer data. Taking the anemometer results as the ground truth, the STDs of wind direction and speed using single-curvefitting are found to be $8.3^{\circ}$ and $0.9 \mathrm{~m} / \mathrm{s}$, respectively, and those for dual-curve-fitting are, respectively, $6.6^{\circ}$ and $0.8 \mathrm{~m} / \mathrm{s}$ (see Table II). It is known that the sea surface roughness increases with wind speed. The buoy-measured significant wave heights are plotted in Fig. 6(c) for illustration. As shown in Fig. 6(a), improvement using dual-curve-fitting is seen during 01:12 to 02:22 when the sea states were low (significant wave height lower than $2.30 \mathrm{~m}$ ). During this period, based on the low-clutter direction percentage analysis, the percentage of directions with many low-intensity pixels is above 50\% [see Fig. 6(c)]. Within this 70-min period, the STDs of wind direction and speed using single-curve-fitting are $3.4^{\circ}$ and $0.2 \mathrm{~m} / \mathrm{s}$, respectively, and those for dual-curve-fitting are, respectively, $3.5^{\circ}$ and $0.2 \mathrm{~m} / \mathrm{s}$. Although the STDs are almost the same, the mean errors of wind direction and speed have been improved by about $5.7^{\circ}$ and $0.3 \mathrm{~m} / \mathrm{s}$ during low sea states. The performances of the single- and dual-curve-fitting techniques are almost the same when the wind speed is higher than $4 \mathrm{~m} / \mathrm{s}$, under which condition the clutter is strong in almost all azimuthal directions [see Fig. 6(b) and (c)].

\section{B. Original ILS Versus Modified ILS}

Fig. 7 shows the results with and without island recognition from Dataset 4, during 17:46 to 18:46, Oct. 29, 2008, which involves the period when ship approached and then left the island from 17:56 to $18: 16$. The island was located in the near range from 18:00 to 18:10 when the results using island recognition show some improvement (the STDs of retrieved wind direction and speed are reduced by about $7^{\circ}$ and $0.3 \mathrm{~m} / \mathrm{s}$, respectively, from those measured by the anemometer, as shown in Table II). It may be observed that from 17:46 to 17:58 and $18: 12$ to $18: 46$, the wind results with island recognition are exactly the same as those without island recognition. This is because the island was located far from the ship during these periods, and the intensity level selection algorithm itself can withstand the island interference automatically. Also, it can be seen that the results using the modified method are more stable than those obtained using the original method. 

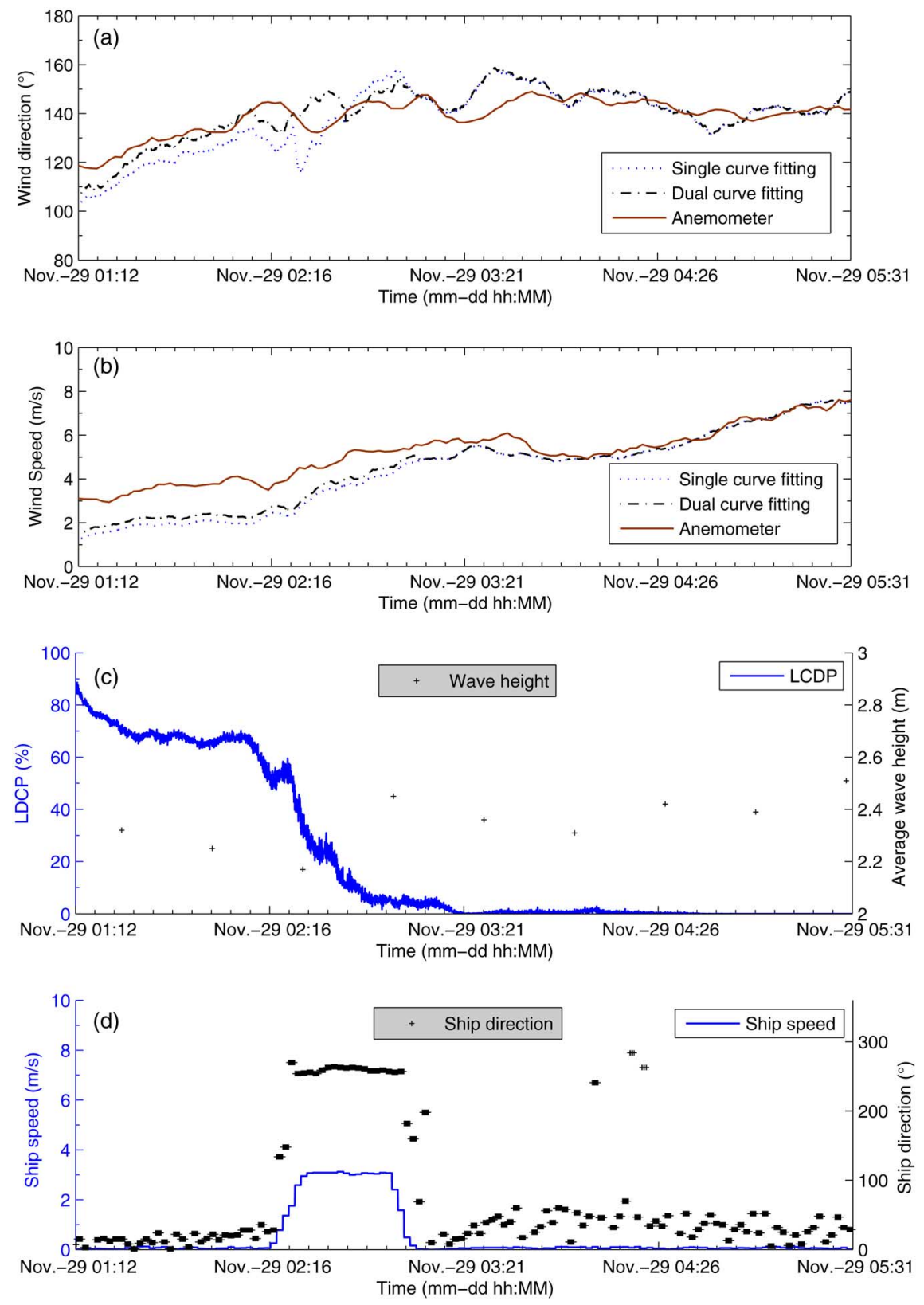

Fig. 6. Comparison of wind results from 01:12 to 05:31, November 29, 2008: (a) wind direction; (b) wind speed; (c) significant wave height and percentage of directions with many low-intensity pixels; and (d) ship speed and ship direction.

Next, the modified ILS method above was applied to a longer dataset of quality-controlled Furuno radar data and the results were compared with the reference data measured by a shipborne anemometer (with the ship's motion removed). Fig. 8 shows the comparison of the radar-retrieved wind velocities obtained from the original and modified ILS-based methods with the anemometer-measured results based on Dataset 2 collected during the period from 12:09, Dec. 1 to 12:02, Dec. 4, in 2008. It may be observed that the radar results from both methods agree well with the anemometer data. However, the results using the modified method are clearly more stable with respect to the wind direction results even when more than half of the image data are discarded due to blockage and low wind speed from 06:30 to 07:30, Dec. 2 [see Fig. 8(a)]. From 00:30 to $06: 00$, Dec. 3, the low-clutter direction percentage is higher than $90 \%$, so those images are considered as low-backscatter cases and are discarded. Moreover, influence of frequent ship motion on the anemometer-measured results is observed at time around 05:00 and 15:00, Dec. 3 in Fig. 8(a). The anemometer winds appear more spiky than the radar results. From Fig. 8(d), it can be seen that the ship motion changed significantly and frequently. For all the Furuno data collected in December, it is shown that the modified ILS-based method reduces the mean differences and STDs between the radar and anemometer results for wind direction and speed by about $3^{\circ}$ and $4.9^{\circ}$ and 0.5 and $0.2 \mathrm{~m} / \mathrm{s}$, respectively (see Table II). 

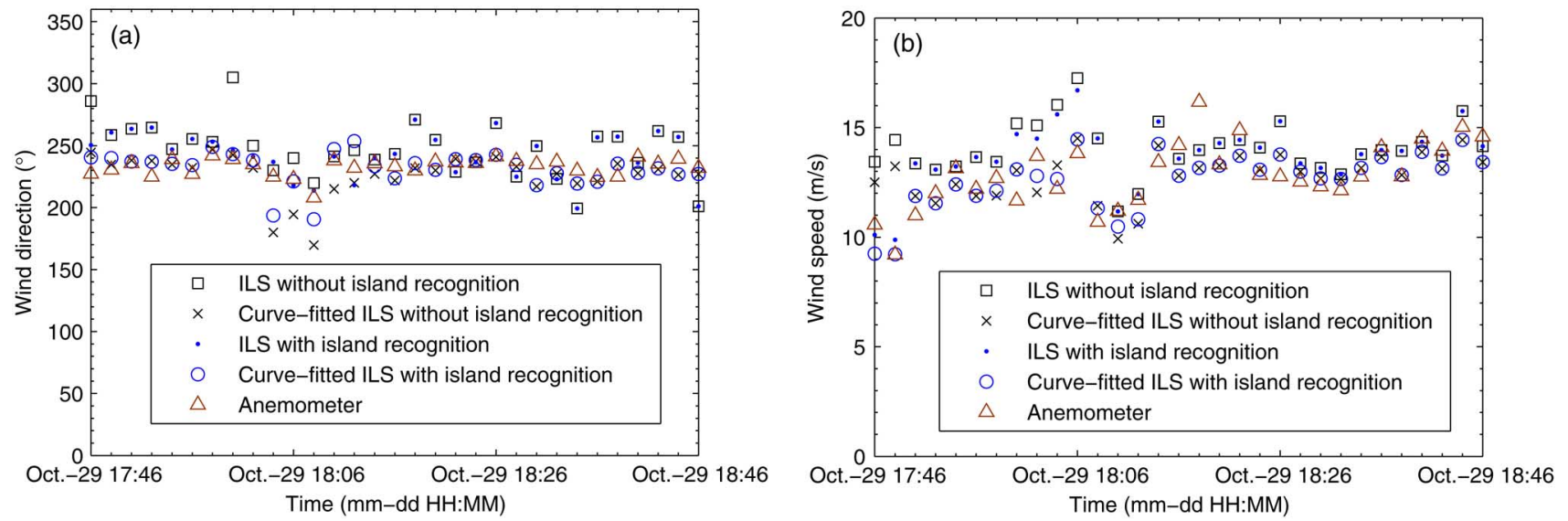

Fig. 7. Comparison results obtained from data (Dataset 4) with appearance of island: (a) wind direction and (b) wind speed.
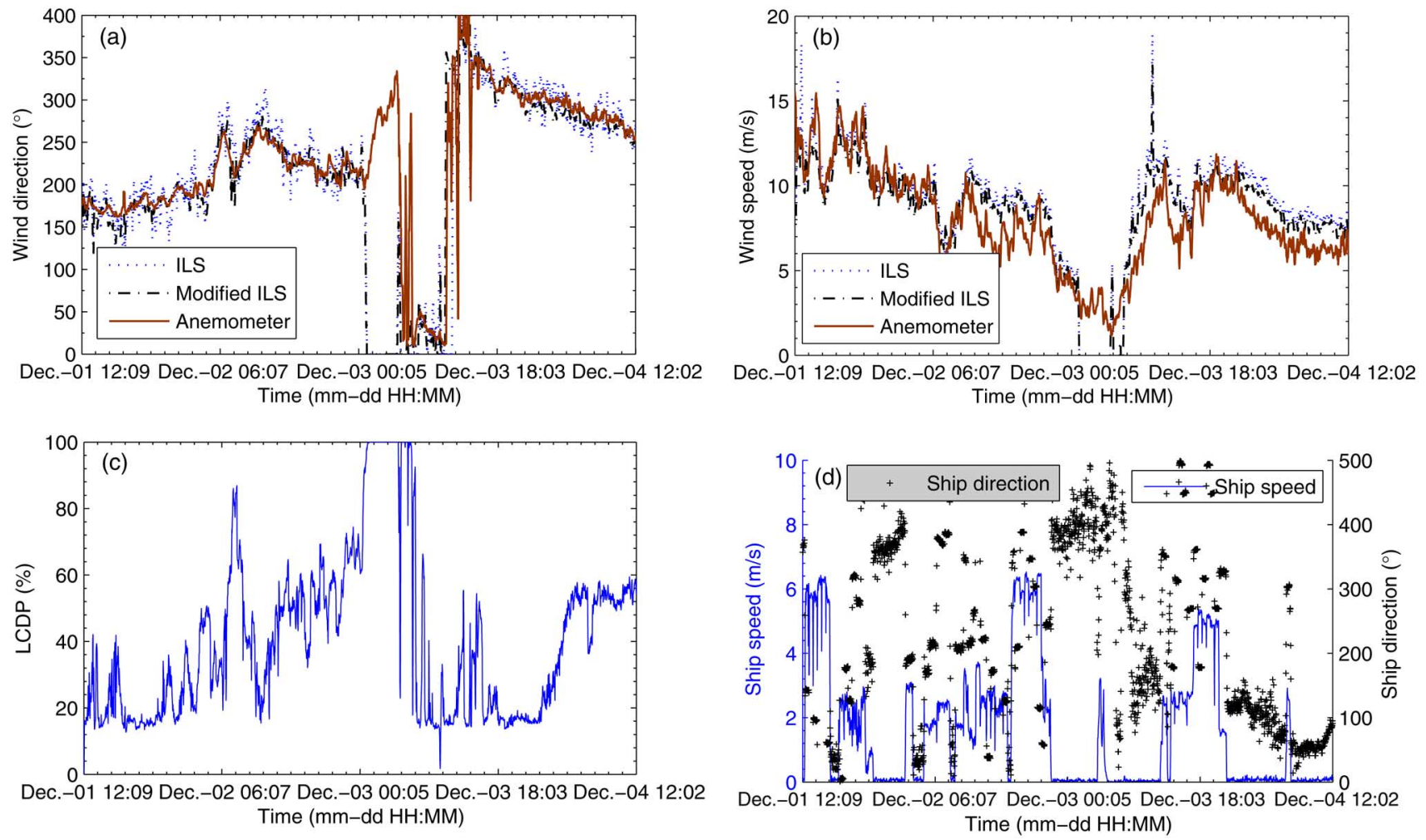

Fig. 8. Comparison of Dataset 2 wind results using ILS and modified ILS from 12:09, Dec. 1 to 12:02, Dec. 4, in 2008: (a) wind direction; (b) wind speed; (c) percentage of directions with many low-intensity pixels; and (d) ship speed and ship direction.

\section{Dual-Curve-Fitting Versus Modified ILS}

By comparing the wind parameter results obtained from Dataset 1 using the modified ILS-based and dual-curve-fitting algorithms with the anemometer data, it may be observed from Fig. 9 that both algorithms can provide satisfactory results in most cases. When the zero pixel percentage is lower than $10 \%$ [see Fig. 9(d)] in this dataset, the data are classified as rain cases. Rain was recognized during $02: 10$ to $05: 40$ and $08: 10$ to $13: 30$ on Nov. $27,18: 00$ to $19: 30$ on Nov. 28 , and $06: 40$ to 09:10 on Nov. 29, when the STDs and mean errors of wind speed and direction between the reference data and the retrieved wind results using both methods are larger than those obtained under the rain-free condition. It was also found that when rain interference exists, the modified ILS method seems more robust than the dual-curve-fitting method for the data considered here. For example, during 11:40 to 12:06, Nov. 29, the wind speed by dual-curve-fitting is significantly overestimated due to rain, while the result from the modified ILS method is satisfactory. Similar conclusions can be made for the period of 08:00 to 10:00, Nov. 27. This may be because, based on our calibration model (see Fig. 2) for the curve-fitting method, a small increase in intensity will cause a relative large increase in wind speed when the speeds are within $13-20 \mathrm{~m} / \mathrm{s}$. However, the ILS-based model looks less sensitive to intensity change over that speed range. A better model with more training data at high wind speeds may improve the wind results obtained from 

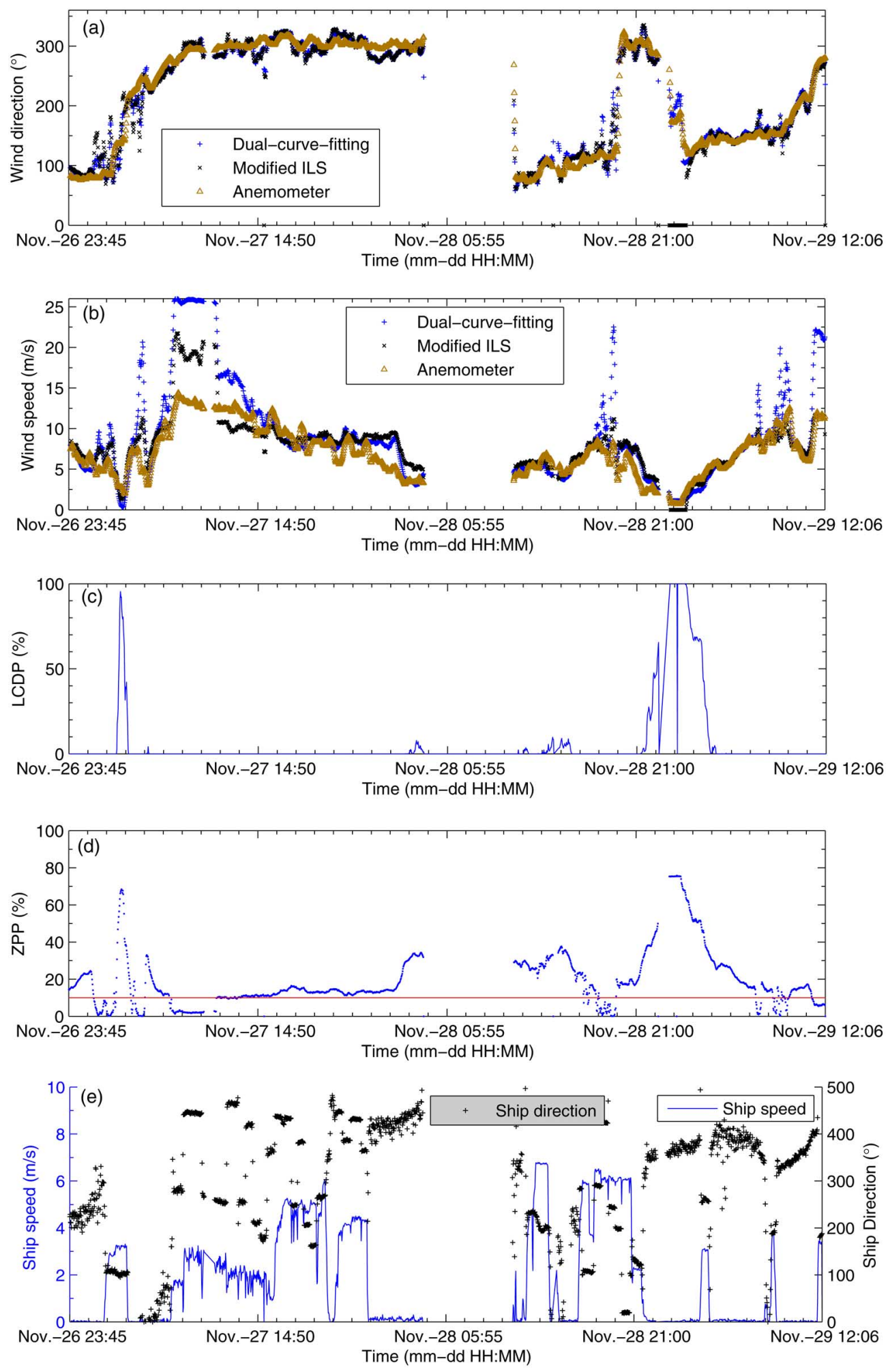

Fig. 9. Comparison results of (a) wind speed; (b) wind direction; (c) low-clutter direction percentage; (d) zero pixel percentage; and (e) ship information for Dataset 1.

the curve-fitting-based method. On the other hand, dual-curvefitting shows superiority over the modified ILS method for wind direction when the low-clutter direction percentage is high due to low sea state [see the peak during 22:16, Nov. 28 to $01: 13$, Nov. 29 in Fig. 9(c)]. The average execution times for processing a set of 32 radar images using dual-curve-fitting and the modified ILS algorithm are 6.001 and $21.919 \mathrm{~s}$, respectively, running in MATLAB on an i5-3450 CPU of $3.10 \mathrm{GHz}$. On the other hand, the least time for collecting a set of 32 radar images is $48 \mathrm{~s}$ (for Dataset 2 or 4). Thus, it can be concluded that the modified approaches may be executed in near real time. The ILS-based algorithms take more time due to the 
interpolation which is implemented to standardize the image size before temporal integration for our datasets. This procedure is not required in the curve-fitting based algorithms.

\section{CONCLUSION}

In this paper, two improved algorithms for wind retrieval from shipborne X-band nautical radar images were proposed and tested using radar and anemometer data collected on the Canadian East Coast. Reference curve-fitting-based and ILSbased algorithms perform satisfactorily. With the improved dual-curve-fitting algorithm, the wind direction and speed mean difference between the radar and anemometer results can be reduced by about $5.7^{\circ}$ and $0.3 \mathrm{~m} / \mathrm{s}$, respectively, under low sea states. However, the results obtained from single- and dualcurve-fitting are almost the same at higher sea states. It is also shown that the modified ILS method (improved algorithm) is more robust than the original ILS-based method (reference algorithm), since the former can work well even when blockages or islands exist in the radar field of view. Although wind speed results extracted from the modified ILS method are not improved distinctly, the modified ILS method reduces the mean differences and STD of wind direction between the radar and the anemometer results significantly by about $3^{\circ}$ and $4.9^{\circ}$ in Dataset 2. Moreover, the radar measurements from dual-curvefitting and the modified ILS agree with each other for most of the data used here, and the mean difference and STD between the anemometer data and the radar results observed using the two methods for wind direction and speed are close. For the data considered in this paper, the overall STDs for wind direction and speed are $6.6^{\circ}-21.2^{\circ}$ and $0.5-2.0 \mathrm{~m} / \mathrm{s}$, respectively, which are a little higher than the results (less than $17.4^{\circ}$ for direction and less than $1.1 \mathrm{~m} / \mathrm{s}$ for speed) in open literatures (e.g., [6]-[9], [22]). It should be noted that the temporally averaged radar-derived wind results also represent spatial average values within the radar coverage, while the temporally averaged anemometer data represent point measurements at the ship location. This may account for some of the difference between the radar and anemometer results. In addition, correcting the wind speed measurements for the atmospheric stability condition may improve the results [22]. However, this procedure is not incorporated here since some necessary parameters for the correction are not available.

A couple of limitations of the methods should be noted. First, modeling of the curve-fitting-based approach is not yet robust enough. Building proper models for wind speed retrieval depends on several factors, such as the accuracy of anemometer data. Second, rain recognition was not successful for some datasets. A more intelligent way to identify and eliminate the effects of rain is required. A threshold in terms of signal-to-noise ratio instead of the gray scale intensity may be used for rain recognition. Furthermore, images in the presence of rain were discarded before wind speed and direction were retrieved in previous work. Further efforts are ongoing to design a method that may be used to recover wind parameters by exploiting the differences between images collected with and without rain. It would also be worthwhile to compare the performance of wind extraction from the horizontally and vertically polarized radar data presented in [23] and [24].

\section{ACKNOWLEDGMENT}

The authors would like to thank Dr. E. Thornhill of Defence Research and Development Canada for providing the radar and buoy data. They would also like to appreciate the five anonymous reviewers for their constructive suggestions to improve the manuscript.

\section{REFERENCES}

[1] B. I. Moat, M. J. Yelland, R. W. Pascal, and A. F. Molland, "The effect of ship shape and anemometer location on wind speed measurements obtained from ships," in Proc. Int. Conf. Marine Comput. Fluid Dynam., Southampton, U.K., Mar. 2005, pp. 133-139.

[2] B. Lund, "Development and evaluation of new algorithms for the retrieval of wind and internal wave parameters from shipborne marine radar data," Ph.D. dissertation, Rosenstiel School of Marine and Atmospheric Science, Univ. Miami, 2012.

[3] P. H. Y. Lee et al., "X-band microwave backscattering from ocean waves," J. Geophys. Res., vol. 100, no. C2, pp. 2591-2611, Feb. 1995.

[4] H. Hatten, F. Ziemer, J. Seemann, and J. Nieto-Borge, "Correlation between the spectral background noise of a nautical radar and the wind vector," in Proc. 17th Int. Conf. Offshore Mech. Arctic Eng. (OMAE), Lisbon, Portugal, Jun. 1998.

[5] D. Trizna and D. Carlson, "Studies of dual polarized low grazing angle radar sea scatter in nearshore regions" IEEE Trans. Geosci. Remote Sens., vol. 34, no. 3, pp. 747-757, May 1996.

[6] H. Dankert, J. Horstmann, and W. Rosenthal, "Wind- and wave-field measurements using marine X-band radar-image sequences," IEEE J. Ocean. Eng., vol. 30, no. 3, pp. 534-542, Jul. 2005.

[7] H. Dankert, J. Horstmann, and W. Rosenthal, "Ocean wind fields retrieved from radar-image sequences," J. Geophys. Res.: Oceans, vol. 108, no. C11, pp. 16-1-16-11, Nov. 2003.

[8] B. Lund, H. C. Graber, and R. Romeiser, "Wind retrieval from shipborne nautical X-band radar data," IEEE. Trans. Geosci. Remote Sens., vol. 50, no. 10 , pp. 3800-3811, Oct. 2012.

[9] R. Vicen-Bueno, J. Horstmann, E. Terril, T. de Paolo, and J. Dannenberg, "Real-time ocean wind vector retrieval from marine radar image sequences acquired at grazing angle," J. Atmos. Ocean. Technol., vol. 30, no. 1, pp. 127-139, Jan. 2013.

[10] B. Lund, H. C. Graber, J. Horstmann, and E. Terrill, "Ocean surface wind retrieval from stationary and moving platform marine radar data," in Proc. IEEE Geosci. Remote Sens. Soc. (IGARSS), Munich, Germany, Jul. 2012, pp. 2790-2793.

[11] Y. Liu, W. Huang, E. W. Gill, and D. Peters, "Dual-curve-fitting based wind retrieval from shipborne nautical X-band radar data," in Proc. MTS/IEEE OCEANS, Taipei, Taiwan, Apr. 2014.

[12] Y. Liu, W. Huang, and E. W. Gill, "Intensity-level-selection-based wind retrieval from shipborne nautical X-band radar data," in Proc. IEEE Geosci. Remote Sens. Soc. (IGARSS), Quebéc, QC, Canada, Jul. 2014, pp. 4386-4389.

[13] (2012, May). WaMoS II Wave and Surface Current Monitoring System Operating Manual Version 4.0 [Online]. Available: http:// oceanwaves.org

[14] D. C. Stredulinsky and E. M. Thornhill, "Ship motion and wave radar data fusion for shipboard wave measurement," J. Ship Res., vol. 55, no. 2, pp. 73-85, Jun. 2011.

[15] W. Huang, E. W. Gill, and J. An, "Iterative least-squares-based wave measurement using X-band nautical radar," IET Radar Sonar Navig., 2014, to be published.

[16] J. Tournadre and Y. Quilfen, "Impact of rain cell on scatterometer data: 1. Theory and modeling," J. Geophys. Res., vol. 108, no. C7, pp. 18-118-14, Jul. 2003.

[17] R. F. Contreras and W. J. Plant, "Surface effect of rain on microwave backscatter from the ocean: Measurements and modeling," J. Geophys. Res., vol. 111, no. C8, p. C08019, Aug. 2006.

[18] C. Melsheimer, W. Alpers, and M. Gade, "Investigation of multifrequency/multipolarization radar signatures of rain cells over the ocean using SIR-C/X-SAR data," J. Geophys. Res., vol. 103, no. C9, pp. 1886718884, Aug. 1998. 
[19] Y. Liu, W. Huang, E. W. Gill, and D. Peters, "Wind direction retrieval from shipborne nautical X-band radar data," in Proc. IEEE Newfoundland Electr. Comput. Eng. Conf. (NECEC), St. John's, Canada, Nov. 2013.

[20] W. J. Plant, "A two-scale model of short wind-generated waves and scatterometry," J. Geophys. Res., vol. 91, no. C9, pp. 10735-10759, Sep. 1986.

[21] H. Hatten, J. Seemann, J. Horstmann, and F. Ziemer, "Azimuthal dependence of the radar cross section and the spectral background noise of a nautical radar at grazing incidence," in Proc. IEEE Geosci. Remote Sens. Soc. (IGARSS), vol. 5, Seattle, WA, USA, Jul. 1998, pp. 2490-2492.

[22] H. Dankert and J. Horstmann, "A marine radar wind sensor," J. Atmos. Ocean. Technol., vol. 24, no. 9, pp. 1629-1642, Sep. 2007.

[23] W. Huang and E. W. Gill, "Surface current measurement under low sea state using dual polarized X-band nautical radar," IEEE J. Sel. Topics Appl. Earth Observ. Remote Sens., vol. 5, no. 6, pp. 1868-1873, Dec. 2012.

[24] J. An, W. Huang, and E. W. Gill, "A self-adaptive wavelet-based algorithm for wave measurement using nautical radar," IEEE Trans. Geosci. Remote Sens., vol. 53, no. 1, pp. 567-577, Jan. 2015.

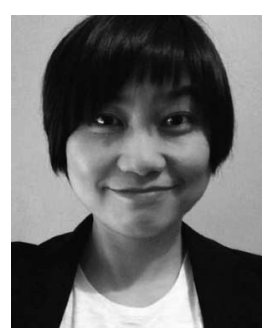

Ying Liu (S'12) was born in Yanshi, China. She received the B.Eng. degree in electronic science and technology from Wuhan University, Wuhan, China, in 2011. Currently, she is pursuing the M.Eng. degree in electrical engineering at Memorial University, St. John's, NL, Canada.

Her research interests include maritime navigation radar wind algorithm development.

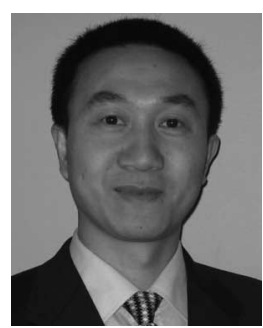

Weimin Huang (M'10-SM'13) received the B.S., M.S., and Ph.D. degrees in radio physics from Wuhan University, Wuhan, China, in 1995, 1997, and 2001, respectively, and the M.Eng. degree and the Postdoctoral Fellowship in engineering electromagnetics from Memorial University, St. John's, NL, Canada.

From 2008 to 2010, he worked as a Design Engineer at Rutter Technologies, St. John's, NL, Canada. Since 2010, he has been an Assistant Professor with the Faculty of Engineering and Applied Science, Memorial University. His research interests include the mapping of oceanic surface parameters via HF ground wave radar and, more recently, target detection and tracking in ocean clutter using marine radar.

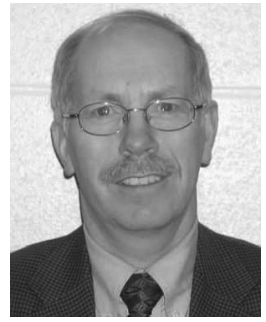

Eric W. Gill (M'00-SM'05) received the B.Sc. degree in physics and the M.Eng. and Ph.D. degrees in electrical engineering from Memorial University, St. John's, NL, Canada, in 1977, 1990, and 1999, respectively.

In 1977, he was a Lecturer in physics with the College of the North Atlantic (formerly, Cabot Institute of Applied Arts and Technology), St. John's, NL, Canada. Since 2000, he has been working with the Faculty of Engineering and Applied Science, Memorial University, where he is currently a Professor, carrying out teaching and research in theoretical and applied electromagnetics. His research interests include the scattering of high-frequency electromagnetic radiation from time-varying, randomly rough surfaces, with particular application to the use of ground wave radar in remote sensing of the marine environment.

Prof. Gill is a member of the American Geophysical Union.

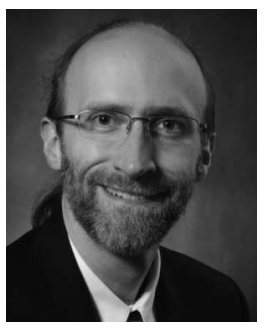

Dennis K. Peters (M'86-SM'06) received the B.Eng. (electrical) degree from Memorial University, St. John's, NL, Canada, in 1990, and the M.Eng. and $\mathrm{Ph} . \mathrm{D}$. degrees in electrical and computer engineering from McMaster University, Hamilton, ON, Canada, in 1995 and 2000, respectively.

$\mathrm{He}$ is an Associate Professor and Department Head of Electrical and Computer Engineering with Memorial University, where he has been since 1998. His research interests include techniques for design and verification of software and computer systems, with particular focus on high-performance computing and parallel and distributed processing, and software engineering education.

Dr. Peter is a licensed Professional Engineer in the province of Newfoundland and Labrador and a member of the ACM and the ACM SIGSOFT.

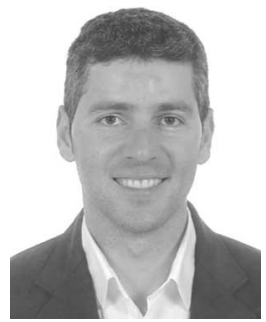

Raul Vicen-Bueno received the B.Eng/B.Sc. and M.Sc. degrees in electrical engineering in 2000 and 2002, respectively, and the Ph.D. degree in electrical engineering in 2012, all from the University of Alcala (UAH), Madrid, Spain.

From 2000 to 2002, he worked as a Research and Development Engineer with INDRA, S.A., Madrid, Spain. In 2002, he joined as a Researcher with the Signal Theory and Communications Department, UAH, where he holds an Associate Professor position now. He was a Visiting Scientist with the University of Chalmers, Gothenburg, Sweden, in 2007, and NATO Undersea Research Centre, NURC, La Spezia, Italy, in 2010 and 2011. From 2013, he works as a Project Leader with NATO STO Centre for Maritime Research and Experimentation, CMRE, La Spezia, Italy. His research interests include decision support systems, remote sensing, pattern recognition, artificial intelligence, optimization, and signal processing, mainly focused on the maritime domain. 